\section{La erradicación de la malaria en Puerto Rico'}

\author{
Rafael Miranda Franco ${ }^{2} y$ \\ Alfredo Casta Vélez ${ }^{3}$
}

La malaria ya era conocida antes de la era cristiana. Aunque se ignoraba su modo de transmisión, la enfermedad solía atribuirse al "aire malo" de pantanos y aguas estancadas, de donde surgió su nombre. En el siglo XIX finalmente se descubrió que un parásito del género Plasmodium era el agente causal de la malaria y más tarde que sus vectores eran los mosquitos del género Anopheles. Por su situación geográfica, Puerto Rico reúne todas las condiciones necesarias para la transmisión de la malaria: clima cálido y húmedo, abundantes criaderos del mosquito vector (Anopheles albimanus) y una población susceptible a la enfermedad, que es endémica en casi todo el territorio, salvo en las alturas del interior.

Debido al desconocimiento del modo de transmisión, antes de comienzos de este siglo se había hecho poco para prevenir y controlar la enfermedad. En 1911 las autoridades sanitarias, preocupadas por los muchos casos de malaria y la abundante población de mosquitos en la ciudad de San Juan, recomendaron rellenar los manglares en las llanuras y a orillas de la bahía. En 1918 se firmó un convenio con la Fundación Rockefeller para recibir asesoramiento sobre investigaciones para la prevención y el control de la malaria y otras enfermedades transmisibles. Finalmente, el Negociado de Control de Malaria del Departamento de Sanidad (hoy de Salud) de Puerto Rico se creó en 1925, fecha en que se iniciaron las actividades de control en el país. En aquel momento la enfermedad atacaba mayormente a la población rural, que hasta la década de 1950 era la más numerosa (cuadro 1).

\section{LAS ACTIVIDADES DE CONTROL}

\section{Primera fase (1925-1940)}

El programa se inició en la ciudad de Fajardo en 1925, en un radio de acción de 3,2 km desde la población bajo control. Las medidas utilizadas consistían en el tratamiento de enfermos con quinina; zanjeo y drenaje de aguas estancadas, relleno de tierras donde fuera posible, y aplicación de verde de París como larvicida para reducir el número de criaderos. Posteriormente el programa se extendió a otras poblaciones con una alta incidencia de la enfermedad, entre ellas Salinas, Santa Isabel, Guayama, Patillas, Arroyo, San Germán, Yabucoa y Ponce.
Gran parte del presente trabajo se basa en: Palacios LD. Informe sobre la lucha antimalárica en Puerto Rico. San Juan: Departamento de Salud; 1961.

2 Ex funcionario de la Organización Panamericana de la Salud, Washington, DC, Estados Unidos de América. Dirección postal: 524 Calle José R. Acosta, San Juan, Puerto Rico 00918-2847.

3 Consultor, Departamento de Salud, San Juan, Puerto Rico. 
CUADRO 1. Población de Puerto Rico, según los censos de 1930 a 1990

\begin{tabular}{lccccc}
\hline Año & $\begin{array}{c}\text { Población } \\
\text { total }\end{array}$ & $\begin{array}{c}\text { Población } \\
\text { urbana }\end{array}$ & $\%$ & $\begin{array}{c}\text { Población } \\
\text { rural }\end{array}$ & $\%$ \\
\hline 1930 & 1543913 & 427221 & 28 & 1116692 & 72 \\
1940 & 1869225 & 566357 & 30 & 1302898 & 70 \\
1950 & 2210703 & 894913 & 40 & 1315890 & 60 \\
1960 & 2349544 & 1039301 & 44 & 1310243 & 56 \\
1970 & 2712033 & 1575491 & 58 & 1136542 & 42 \\
1980 & 3196530 & 2134365 & 67 & 1062155 & 33 \\
1990 & 3522037 & 2508346 & 71 & 1013691 & 29 \\
\hline
\end{tabular}

Fuente: Junta de Planificación de Puerto Rico, Oficina del Censo.

La eficacia de las actividades de control se medía mediante la captura de mosquitos adultos con trampas especiales y el uso de animales como cebo; la inspección de los criaderos para determinar la densidad larvaria; y la toma de muestras de sangre obtenidas de la población a fin de conocer el índice de positividad al parásito. Los primeros resultados no fueron muy satisfactorios y apenas se notaba una pequeña reducción de casos en las áreas protegidas. Pero como la enfermedad continuaba haciendo estragos en el resto del país, fue necesario emplear otras medidas, optándose por obras de ingeniería que eliminaran permanentemente los criaderos. Para este fin en 1928 la Fundación Rockefeller otorgó una beca al ingeniero Luis D. Palacios para que se especializara en drenajes con fines agrícolas y sanitarios en la Universidad de Cornell en los Estados Unidos.

A comienzos de los años treinta, tras una serie de experiencias en varias áreas, se realizaron en Salinas las primeras obras de drenaje. Debido al alto costo de las mismas, se solicitó la cooperación económica de los municipios y de los grandes terratenientes en áreas con una alta incidencia de malaria, puesto que la medida no solo resolvía el problema de absentismo entre los trabajadores, sino que permitía recuperar terrenos baldíos para la agricultura. Tales acciones estimularon la participación de otros terratenientes. Por otro lado, era la época de la gran depresión económica y Puerto Rico se benefició del programa New Deal ("Nuevo Trato") establecido por el presidente Franklin Delano Roosevelt con fondos para la generación de empleo. Parte de esos fondos fueron dedicados a recuperar terrenos pantanosos e inundables mediante la realización de obras de ingeniería antimalárica.

En los albores de la Segunda Guerra Mundial, también con fondos federales e insulares, se contrató para la búsqueda y tratamiento de casos a trabajadores jóvenes que además colaboraban en las obras de drenaje. Estas consistían en a) drenaje por gravedad de terrenos húmedos y relativamente altos, usando tuberías de hormigón soterradas; b) drenaje de terrenos húmedos y bajos con tubería soterrada y bombeo; c) drenaje de pantanos y manglares por bombeo; d) relleno de pequeñas lagunas y charcas; e) introducción de agua de mar en pantanos con agua dulce, y f) revestimiento de cauces de quebradas con medios tubos de hormigón.

Durante la primera fase del programa de control (1925-1940) no se logró reducir de forma importante la incidencia de la enfermedad (cuadro 2). Algunos acontecimientos adversos, tales como los huracanes San Felipe en 1928 y San Ciprián en 1932, así como la falta de recursos debido a la depresión y algunas deficiencias administrativas, afectaron adversamente al programa. Ya para fines del período, después del inicio de la Segunda Guerra Mundial y de la reorganización del Negociado de Control de Malaria, se observó una mejoría que permitió intensificar la búsqueda de enfermos y lograr su cura radical con tratamientos a base de quinina, atebrina, euquinina y plasmoquina.

CUADRO 2. Casos de malaria y defunciones por esta causa. Puerto Rico, 1928 a 1955

\begin{tabular}{|c|c|c|c|c|}
\hline Año & Casos & $\begin{array}{l}\text { Tasa de } \\
\text { morbilidad }^{a}\end{array}$ & Defunciones & $\begin{array}{c}\text { Tasa de } \\
\text { mortalidad }\end{array}$ \\
\hline 1928 & 29888 & 1989,6 & 2166 & 144,8 \\
\hline 1929 & 17471 & 1144,9 & 2108 & 137,0 \\
\hline 1930 & 21733 & 1402,1 & 1887 & 121,7 \\
\hline 1931 & 47656 & 3027,6 & 3208 & 203,7 \\
\hline 1932 & 46045 & 2879,4 & 2797 & 174,9 \\
\hline 1933 & 45613 & 2089,2 & 3259 & 200,7 \\
\hline 1934 & 24049 & 1450,6 & 2518 & 152,8 \\
\hline 1935 & 13975 & 838,4 & 2112 & 126,2 \\
\hline 1936 & 14416 & 849,2 & 2401 & 141,4 \\
\hline 1937 & 26045 & 1468,2 & 2296 & 129,8 \\
\hline 1938 & 35659 & 1975,6 & 1962 & 100,7 \\
\hline 1939 & 24101 & 1312,2 & 1637 & 89,1 \\
\hline 1940 & 23578 & 1261,5 & 1817 & 97,0 \\
\hline 1941 & 21391 & 1228,7 & 2302 & 124,0 \\
\hline 1942 & 21391 & 1009,8 & 1933 & 91,4 \\
\hline 1943 & 16032 & 810,3 & 1166 & 58,9 \\
\hline 1944 & 11209 & 551,1 & 1044 & 49,9 \\
\hline 1945 & 6168 & 301,6 & 881 & 43,1 \\
\hline 1946 & 5584 & 263,5 & 672 & 32,3 \\
\hline 1947 & 3915 & 184,3 & 425 & 20,1 \\
\hline 1948 & 1299 & 60,5 & 253 & 11,8 \\
\hline 1949 & 353 & 16,2 & 90 & 4,2 \\
\hline 1950 & 73 & 3,3 & 57 & 2,6 \\
\hline 1951 & 88 & 3,9 & 32 & 1,6 \\
\hline 1952 & 134 & 5,9 & 13 & 0,6 \\
\hline 1953 & 28 & 1,2 & 2 & 0,1 \\
\hline 1954 & 13 & 0,6 & 3 & 0,13 \\
\hline 1955 & 7 & 0,3 & 0 & 0,00 \\
\hline
\end{tabular}

Fuente: Palacios LD. Informe sobre la lucha antimalárica en Puerto Rico. San Juan: Departamento de Salud; 1961. 


\section{Segunda fase (1941-1955)}

Irónicamente, la Segunda Guerra Mundial tuvo un efecto beneficioso en la campaña de erradicación de la malaria en Puerto Rico. Aunque los Estados Unidos de América entraron al conflicto bélico en 1941, ya en 1940 se había comenzado a ampliar las bases militares y a construir bases nuevas en lugares estratégicos, como Puerto Rico en la zona del Caribe. Constituyen ejemplos la base naval de Roosevelt Roads en Ceiba y la base aérea Ramey Field en Aguadilla. Debido a que muchas de estas instalaciones militares estaban en áreas con alta incidencia de malaria, el gobierno de los Estados Unidos asignó fondos especiales para el control de la enfermedad. Puerto Rico hizo otro tanto, gracias al programa gubernamental de 1940 encaminado a mejorar las condiciones socioeconómicas del país, cuyas prioridades eran el desarrollo industrial, la agricultura, la salud y la educación. Como consecuencia de ello se reorganizó el Negociado de Control de Malaria bajo la dirección de un ingeniero sanitario.

Simultáneamente los Estados Unidos, impulsados por el problema de la malaria en el sur del país, crearon la Agencia para el Control de la Malaria en Zonas Bélicas (Malaria Control in War Areas, o MCWA), dependencia del Servicio de Salud Pública (United States Public Health Service, USPHS), para que se hiciera cargo del control de la malaria en lugares adyacentes a las bases militares. Esta unidad inició sus actividades en Puerto Rico en coordinación con el Negociado de Control de Malaria. Pese a la escasez de materiales y equipo ocasionada por la guerra, se construyó el "Canal de la Malaria" en Cataño con objeto de drenar terrenos bajos y húmedos en las proximidades del Fuerte Buchanan. Con el fin de reducir la población de mosquitos adultos se fumigaron las casas vecinas con insecticidas piretroides y se realizaron obras de drenaje cerca de los campamentos de Tortuguero (Vega Baja), Losey Field (Juana Díaz) y Roosevelt Roads (Ceiba).

En 1944, como parte de los trabajos de investigación efectuados por el USPHS con la colaboración de la Escuela de Medicina Tropical y del Departamento de Salud de Puerto Rico, se comenzó a utilizar el rociamiento intradomiciliario con diclorodifeniltricloroetano (DDT) de forma experimental. Las primeras pruebas se llevaron a cabo en la Playa de Humacas y Santa Isabel. La característica principal de este insecticida era su efecto residual, que duraba de 6 meses a un año. El producto se había utilizado con éxito desde 1942 en los frentes de guerra contra piojos, mosquitos y otros vectores de enfermedades. En 1946 se decidió utilizar el rociamiento con DDT como medida adicional con- tra la malaria en las áreas que hubieran tenido las mayores tasas de incidencia de la enfermedad en los 10 años anteriores. Se comenzó a aplicar DDT en ciclos semestrales en 26 municipios y para principios de los años cincuenta se había rociado casi toda la isla (cuadro 3).

En 1947 se hizo un intento por erradicar Anopheles albimanus de Puerto Rico. Llegó un grupo de eminentes malariólogos (un parasitólogo, un entomólogo y un ingeniero sanitario) para dar asesoramiento sobre la planificación de un programa piloto basado en la eficacia del DDT. Por su magnitud y costos, el programa no se llevó a cabo, aunque a la larga resultó innecesario para erradicar la enfermedad.

Cuando terminó la guerra en 1945, se redujeron las actividades militares en Puerto Rico y en 1946 cesó sus funciones en la isla la Agencia para el Control de la Malaria en Zonas Bélicas. El gobierno local continuó apoyando el programa de control y adjudicó fondos para obras de drenaje en los alrededores de Ponce, Santa Isabel y Salinas, a pesar de la marcada disminución de los casos de malaria. Para fines de la década se descontinuó la aplicación de larvicidas en áreas con pocos casos, manteniéndose como medida principal de control el rociamiento de casas con DDT. Asimismo, se disminuyó la construcción de nuevas obras de drenaje y se dio prioridad a una estrecha vigilancia epidemiológica a fin de detectar todos los casos de malaria y lograr su cura radical.

En la era posterior a la guerra, caracterizada por el desarrollo económico de Puerto Rico, se vislumbraba por primera vez la posibilidad de erradicar la enfermedad de la isla y el Negociado de Control de Malaria pasó a ser el Negociado de Control de Malaria e Insectos. Entre los principales fac-

CUADRO 3. Rociamiento de viviendas con diclorodifeniltricloroetano (DDT) en Puerto Rico, 1946 a 1955

\begin{tabular}{lcc}
\hline Año fiscal $^{\mathrm{a}}$ & Municipios cubiertos (No.) & Rociamientos \\
\hline 1946 & 1 & 2499 \\
$1946-1947$ & 28 & 78249 \\
$1947-1948$ & 30 & 100360 \\
$1948-1949$ & 29 & 92550 \\
$1949-1950$ & 44 & 173250 \\
$1950-1951$ & 57 & 169357 \\
$1951-1952$ & 61 & 167861 \\
$1952-1953$ & 47 & 129330 \\
$1953-1954$ & 18 & 132116 \\
$1954-1955$ & 16 & 74082 \\
\hline
\end{tabular}

Fuente: Palacios LD. Informe sobre la lucha antimalárica en Puerto Rico. San Juan: Departamento de Salud; 1961.

${ }^{a}$ De 1946 a 1950 se realizaron dos ciclos de rociamiento anuales, y uno solo a partir de 1950. De 1952 en adelante se aplicó DDT para el control de la malaria y la erradicación de Aedes aegypti. 
tores que contribuyeron a la reducción de la malaria, además de las medidas de control, cabe mencionar la mayor comercialización de la agricultura y el establecimiento de industrias, que llevaron a un incremento en la construcción, especialmente de viviendas. Con la consecuente disminución de la población rural y el aumento de la población urbana (cuadro 1), en el período de 1945 a 1950 se produjo un brusco descenso de los casos de malaria en el país: de 6168 en 1945 a solo 73 en 1950 (cuadro 2). Esto motivó un nuevo enfoque del Negociado de Control de Malaria e Insectos, entidad que tomó a su cargo la campaña de erradicar Aedes aegypti, vector de la fiebre amarilla y del dengue, como parte de un movimiento continental que se produjo en 1950.

Entre 1951 y 1955, casi todos los casos de malaria registrados correspondían a veteranos de la guerra de Corea. Por tal motivo, las medidas de control se descontinuaron paulatinamente en las áreas sin casos de malaria. En 1954 se juzgó que la enfermedad ya no era un problema de salud pública en Puerto Rico y en 1955 el Negociado de Control de Malaria e Insectos cesó sus actividades y se convirtió en la Sección de Control de Vectores del Negociado de Saneamiento Ambiental.

\section{Tercera fase (1956-1995)}

De 1956 a 1960 solo se registraron 10 casos importados de malaria en Puerto Rico (en 1956, 1; en 1957, 7; en 1958, 1; y en 1959, 1). Con el éxito de la lucha contra la malaria, las iniciativas prioritarias de la Sección de Control de Vectores se concentraron en la erradicación de Aedes aegypti, el control de la esquistosomiasis mediante la aplicación de molusquicidas, el tratamiento de casos, la fabricación y distribución de letrinas sanitarias y el mantenimiento de las obras de drenaje antimalárico. De 1964 a 1969 Puerto Rico contó con el financiamiento del programa de los Estados Unidos para la erradicación de Aedes aegypti, que fue creado para los estados del sur del país, aunque con algún retraso en relación con otros países. A partir de 1969 y de manera limitada, con apoyo económico local, las actividades se concentraron en campañas de control principalmente educativas y comunitarias para eliminar los criaderos de mosquitos.

El primer brote de dengue en la isla se registró en 1963 y un segundo brote tuvo lugar en 1969. Fue así como a partir de 1975 la División de Higienización del Ambiente Físico del Departamento de Salud, que reemplazó a la Sección de Control de Vectores, se hizo cargo de dicho programa, utilizando $65 \%$ de su presupuesto anual para ese propósito y el resto para el control de otros insectos vectores de enfermedades. Por ser una enfermedad de gran impacto sobre la salud pública del país, el dengue es un problema prioritario al que se ha dedicado un sistema de vigilancia epidemiológica de gran calibre. El sistema cuenta con un laboratorio para el estudio exclusivo de casos de dengue que pertenece a los Centros para el Control y la Prevención de Enfermedades del USPHS.

En 1977, 1978, 1981, 1982, 1986 y 1994 se presentaron brotes epidémicos de dengue en la isla, $y$ en 1986 aparecieron los primeros casos de dengue hemorrágico confirmados por el laboratorio, entre ellos tres defunciones. De ahí en adelante los casos han venido aumentando; en 1994 se registraron 143 casos y 12 defunciones.

En mayo de 1986, después de un desastre en Mameyes, cerca de Ponce, causado por las inundaciones en el sur de la isla, la Agencia Federal para Emergencias (Federal Emergency Management Agency) solicitó al gobierno de Puerto Rico que implantara un plan de mitigación para inundaciones en esta área. De ahí surgió un comité interinstitucional de trabajo encargado de poner en práctica el Plan de Acción Zona Sur. El problema más serio, que era la disposición de aguas pluviales, se vio agravado por el mal mantenimiento de los sistemas de drenaje, incluso de los que fueron construidos para el control de la malaria. Con tal motivo se transfirieron los sistemas de bombeo antimaláricos del Departamento de Salud al Departamento de Recursos Naturales y se asignaron los fondos necesarios para rehabilitar las estaciones de bombeo.

De acuerdo con un estudio de la División de Epidemiología del Departamento de Salud, entre 1970 y 1989 se registraron en Puerto Rico 173 casos de malaria, de los cuales todos se consideraron importados. La mayoría de estos casos eran veteranos de la guerra de Vietnam y los restantes venían del Africa, Centroamérica, Haití y República Dominicana.

Desde los años cincuenta no se ha registrado un solo caso autóctono de malaria en Puerto Rico. Esporádicamente aparecen casos en personas que provienen de países de alta incidencia de malaria o que los han visitado. Tan pronto muestran los síntomas, estas personas son tratadas por las autoridades sanitarias del país.

\section{CONCLUSIONES}

La malaria era una de las principales causas de enfermedad y mortalidad en Puerto Rico durante las primeras décadas del presente siglo. En 1925 los trabajos de control se iniciaron en Fajardo $\mathrm{y}$ en años subsiguientes se extendieron a otras zonas de alta incidencia. 
En un principio las medidas de control consistían en la aplicación de larvicidas, la limpieza de criaderos de mosquitos y la distribución de medicamentos antimaláricos. Ya en la década de 1930 se comenzaron algunas obras de ingeniería con el fin de eliminar los criaderos permanentemente. Para fines de esa década, se intensificaron las medidas de control, que cobraron impulso después de la entrada de los Estados Unidos a la Segunda Guerra Mundial en 1941. Durante los años cuarenta se contó con recursos económicos adicionales proporcionados por los gobiernos de Puerto Rico y los Estados Unidos para obras antimaláricas. En 1944 se comenzó a utilizar DDT en el país de forma experimental y en 1946 el rociamiento residual de las viviendas con este insecticida se agregó como medida de control adicional. Simultáneamente se incrementaron la búsqueda y el tratamiento de casos.

A partir de 1942 se comenzó a observar un descenso paulatino de la incidencia de malaria, que se aceleró en la segunda mitad de esa década: de 21391 casos y 1933 defunciones en 1942 las cifras se redujeron a 73 casos y 57 defunciones en 1950 .

A fines de la década de 1940 se vislumbró la posible erradicación de la malaria del país. De ahí en adelante se fueron suspendiendo las medidas de control en áreas con muy baja incidencia, donde se intensificó la vigilancia epidemiológica. La incidencia de la enfermedad siguió bajando aceleradamente y en 1954 se consideró que la malaria ya no constituía un problema de salud pública en Puerto Rico. Casi todos los casos que se presentaron en el período de 1951 a 1954 fueron veteranos que regresaban de la guerra de Corea; los casos restantes habían sido importados de otros países. En vista de este panorama favorable, se solicitó a la OMS que certificara la erradicación de la malaria en Puerto Rico. Este organismo concedió la certificación en 1962.

No cabe duda que factores ajenos a las medidas de control también contribuyeron al éxito obtenido. Entre ellos se destacan el desarrollo económico del país a partir de 1940, el desplazamiento de la población de la zona rural a la urbana, la industrialización y la reducción del cultivo de caña de azúcar.
La campaña para erradicar la malaria de todas partes del mundo, que se inició en 1955, no tuvo el éxito esperado. La resistencia del mosquito vector a los insecticidas y del parásito causal de la enfermedad a los medicamentos antimaláricos, junto con una serie de problemas administrativos y logísticos y la constante movilización de la población, obstaculizaron la campaña, que se suspendió a fines de los años setenta.

A pesar de estos problemas, se logró erradicar la malaria de otros territorios insulares en la zona del Caribe, como Cuba, Jamaica, Trinidad y Tabago y otras Antillas Menores. También se pudo interrumpir la transmisión de zonas extensas del litoral atlántico en el Brasil, de la costa del Caribe en Venezuela y de algunas partes de Costa Rica, México y Panamá.

La enfermedad sigue existiendo principalmente en zonas rurales de difícil acceso donde el mosquito vector y el parásito muestran resistencia y donde la población está en continuo movimiento. En estas zonas se ha producido un recrudecimiento de la malaria en los últimos años. En las Américas la mayor incidencia se registra en la zona amazónica, pero también hay áreas problemáticas en Centroamérica y en el noroeste de Colombia. A pesar de la presencia del vector de la malaria en Puerto Rico, la transmisión de la enfermedad no ha reaparecido en el país.

\section{SYNOPSIS}

\section{The eradication of malaria in Puerto Rico}

For decades, malaria was a serious public health problem in Puerto Rico. In 1962, that country became the first tropical territory in the Americas, and possibly in the world, to receive certification from WHO for having eradicated malaria and having declared itself free of the disease. This report chronicles the tremendous effort that was dedicated to the eradication campaign in Puerto Rico, which took place in the first part of this century. 